\title{
Relating Brownian motion to diffusion with superparamagnetic colloids
}

\author{
A. Darras* \\ GRASP, CESAM - Physics Department, University of Liège, B-4000 Liège, Belgium and \\ F.R.S.-FRNS, B-1000 Bruxelles, Belgium, http://www.fnrs.be \\ J. Fiscina \\ Experimental Physics, Saarland University, D-66123 Saarbrücken, Germany \\ N. Vandewalle and G. Lumay \\ GRASP, CESAM - Physics Department, University of Liège, B-4000 Liège, Belgium
}

(Dated: August 30, 2016)

Abstract

The present paper introduces an original experiment allowing students to relate the Brownian motion of a set of superparamagnetic colloidal particles to their macroscopic diffusion. An external and constant magnetic field is firstly applied on the colloidal suspension so the particles self-organize into chains. When the magnetic field is removed, particles then freely diffuse from their positions in the chain. It is worthwhile to notice that all the particles initially have the same coordinate on the axis perpendicular to the initial chain. This configuration then enables the observer to study the one dimensional diffusion process, while actually seeing the underlying Brownian motion of the microscopic particles. Moreover, by studying the evolution of the particles distribution, a measurement of the diffusion coefficient is performed. By repeating this measurement with fluids of various viscosities, the Stokes-Einstein relation is eventually illustrated.

\section{INTRODUCTION}

Establishing the properties of a macroscopic system from the behavior of its individual microscopic particles is the main principle of statistical physics. A key topic where such relation is achieved is the macroscopic diffusion resulting from Brownian motion of colloidal particles. Indeed, many papers and introductory courses focus on the link between the Brownian motion and the diffusion to introduce statistical physics concepts. ${ }^{1-6}$ The basic principle of most experiments presented in these papers is to track the trajectory of colloidal particles to deduce the diffusion coefficient by measuring the mean-squared displacement along time. However, the tracking of single particles require to apply ready-to-use image analysis softwares with embedded high-level functions. While students can benefit from learning using those kinds of tools, it may also give them the feeling to use black boxes.

Another regular topic of statistical physics is to study the organization of particles due to their mutual interactions. An example of interaction between particle is the magnetic dipoles' interaction, whose description is then pedagogically relevant. This interaction can be observed with superparamagnetic colloids, which are magnetic nanoparticles inserted in a matrix of non-magnetic material (polystyrene or silica) to obtain particles with diameter $d$ ranging from $10^{2} \mathrm{~nm}$ to a few micrometers. These composite particles are combining a quasi-zero remanent magnetization and a high magnetic response. ${ }^{7,8}$ Quasi-zero remanent magnetization means that, as soon as no magnetic field is applied on the particles, they no longer have any non-negligible magnetic moment and then behave as non-magnetic colloids. A high magnetic response implies that if the particles are immersed in a magnetic field they acquire magnetic dipoles with the same order of magnitude as ferromagnetic particles. This last property implies that particles can efficiently self-organize themselves when immersed in an external magnetic field. If this field is constant, the particles form chains aligned with the field. ${ }^{7,9-14}$ Those chains of superparamagnetic colloids are used in a wide range of applications namely protein isolation, cell sep- aration, waste capture, bacteria processing, chromatography, etc $^{7,15-18}$ and are still a matter of current research topic with various configurations of the external magnetic field. ${ }^{14,19-29}$

The objective of this paper is to present an experiment about the diffusion of superparamagnetic colloids, which only requires basic image analysis algorithms to obtain main results. Moreover, exploring the magnetic interactions between those colloids as an example of anisotropic interaction with some analytical model can also educationally benefit the students. The experiment introduced in the present paper is then particularly suitable for introductory lessons of experimental statistical physics and introduces a direct link between the standard topics and some current research area in that field.

\section{BASIC THEORY}

\section{A. Diffusion}

When colloidal particles are immersed in a fluid, they experience interactions with the surrounding molecules of that fluid. Those interactions create a random force acting on the colloidal particle which moves then randomly. They follow a so-called Brownian motion, from the name of Robert Brown who reported it in the literature after observing this behavior with pollen molecules in $1827 .{ }^{30}$ During that motion, it can be shown that the probability density $\rho(\vec{r}, t)$ for the particle to be at position $\vec{r}$ at time $t$ obeys the diffusion law:

$$
\frac{\partial \rho(\vec{r}, t)}{\partial t}=D \Delta_{r} \rho(\vec{r}, t)
$$

where $\Delta_{r}$ is the Laplace operator and $D$ is called the diffusion coefficient. For a perfect sphere, the diffusion coefficient is related to the temperature $T$, the viscosity $\eta$ and the radius of the particle $R$ through the Stokes-Einstein relation

$$
D=\frac{k_{B} T}{6 \pi R \eta}
$$


where $K_{B}$ is the Boltzmann constant. If $n$ is the dimension of the studied system, and if the position $\vec{r}_{0}$ of the particle at time $t_{0}=0$ is known, the initial condition of this equation is $\rho\left(\vec{r}, t_{0}\right)=\delta\left(\vec{r}_{0}\right)$, with $\delta$ the delta function. The solution of the Equation (1) is then, for $t>t_{0}$,

$$
\rho(\vec{r}, t)=\frac{\rho_{0}}{(4 \pi D t)^{n / 2}} \exp -\frac{\left(\vec{r}-\overrightarrow{r_{0}}\right)^{2}}{4 D\left(t-t_{0}\right)} .
$$

The parameter $\rho_{0}$ is equal to one in this case of a single particle. However, when a set of many particles are observed instead of only one, the evolution of their concentration is a realization of the density probability and, in case of an infinite number of particles, the parameter $\rho$ can be related to this concentration. Subsequently, given the chosen units, the concentration may be not normalized. The initial concentration amplitude can then be $\rho_{0} \neq 1$ (with then $\rho\left(\vec{r}, t_{0}\right)=\rho_{0} \delta\left(\vec{r}_{0}\right)$ if all the particles are initially at the same position). However, the concentration of the particles still evolves as described by the Equation (3). Then the random walk of the particles lead to a global spreading of the particles since the Full Width at Half Maximum (FWHM) $W$ of this Gaussian curve evolves as $W=4 \sqrt{\ln (2) D t}$. Experimentally, the difficulty to study a system with a large number of particles, and obeying those laws, comes from obtaining the initial condition $\rho\left(\vec{r}, t_{0}\right)=\rho_{0} \delta\left(\vec{r}_{0}\right)$. In one dimension, this would require to have all the particles aligned along a given axis at some initial time. Fortunately, the interactions between superparamagnetic colloids offer a possibility to achieve this situation.

\section{B. Superparamagnetic colloids}

In a wide range of experimental situations, superparamagnetic colloidal particles can be approximated as spherical dipoles for modeling purposes. ${ }^{7-10,31-39}$ This means their magnetic dipole $\vec{\mu}$ is a sufficient parameter to describe every other magnetic property of a given particle. Indeed, spherical dipole is equivalent to point dipole $^{40}$ and create a magnetic field

$$
\vec{B}_{\mu}=\frac{\mu_{0}}{4 \pi} \frac{3\left(\vec{\mu} \cdot \vec{e}_{r}\right) \vec{e}_{r}-\vec{\mu}}{r^{3}}
$$

at any point $\vec{r}$ if the dipole is at the origin $\vec{r}=\overrightarrow{0}$, with $\mu_{0}$ the magnetic permeability of vacuum and $\vec{e}_{r}=\frac{\vec{r}}{r}$. It is worth to notice that if the dipole is not surrounded by vacuum, this relation remains true if $\mu_{0}$ is replaced by the magnetic permeability of the surrounding medium. However, main diamagnetic media, as water and air, have a magnetic permeability close to $\mu_{0}$. Using $\mu_{0}$ to compute the field in those media is then a good approximation. Any dipole immersed in an external field $\vec{B}(\vec{r})$ and at position $\vec{r}$ also has a magnetic energy $U(\vec{r}, \vec{\mu})=-\vec{\mu} \cdot \vec{B}(\vec{r})$. The potential energy of magnetic interaction between two dipoles $\vec{\mu}_{1}$ and $\vec{\mu}_{2}$ is then given by

$$
U\left(\vec{r}, \vec{\mu}_{1}, \vec{\mu}_{2}\right)=\frac{\mu_{0}}{4 \pi} \frac{\vec{\mu}_{1} \cdot \vec{\mu}_{2}-3\left(\vec{\mu}_{1} \cdot \vec{e}_{r}\right)\left(\vec{\mu}_{2} \cdot \vec{e}_{r}\right)}{r^{3}}
$$

where $\vec{r}$ is then the vector going from the position of the first dipole to the position of the other one.
By definition, in case of (super-)paramagnetic materials, as long as the amplitude of the external magnetic field $\vec{B}=B \vec{e}_{z}$, $\vec{e}_{z}$ being a unitary vector in the direction of $\vec{B}$, is under some saturation value, the dipolar moment of a given particle of radius $R$ can be expressed as

$$
\vec{\mu}=\frac{\chi 4 \pi R^{3} \vec{B}}{3 \mu_{0}},
$$

with $\chi$ the particle susceptibility and $\mu_{0}$ the magnetic permeability of the vacuum. If the magnetic field $\vec{B}$ is over that saturation value, the magnetic susceptibility $\chi$ can not be considered as a constant anymore. From equation (6), we can infer that those particles have a magnetic energy $U_{m} \equiv-\vec{\mu} \cdot \vec{B}=-\frac{\chi 4 \pi R^{3}}{3 \mu_{0}} \vec{B} \cdot \vec{B}$ and all have a magnetic moment in the direction of the field (unless they have a susceptibility $\chi \approx 10$, the field created by the neighboring dipoles has a negligible amplitude in comparison with the external field). Moreover, from equations (6) and (4), and the potential energy of magnetic interaction between two of the particles can then be expressed as

$$
U(r, \theta)=\frac{\chi^{2} 4 \pi R^{6} B^{2}}{9 \mu_{0}}\left(\frac{1-3 \cos ^{2} \theta}{r^{3}}\right),
$$

if $\theta=\arccos \left(\vec{e}_{z} \cdot \vec{e}_{r}\right)$. Two particles then tend to attract each other and to create a pair of particles aligned with the external magnetic field $\vec{B}$, since the minimum value of their interaction energy is reached when $\theta=0$ or $\pi$ and $r$ is minimal (i.e. $r=R$ for solid particles). It is worthwhile to notice that the radial part of the magnetic force

$$
\vec{F}_{r}=-\frac{\partial U}{\partial r} \vec{e}_{r}=\frac{\chi^{2} 4 \pi R^{6} B^{2}}{3 \mu_{0}}\left(\frac{1-3 \cos ^{2} \theta}{r^{4}}\right) \vec{e}_{r}
$$

vanishes if $\theta=\arccos \frac{1}{\sqrt{3}} \approx 55^{\circ}$ or $\theta=\arccos -\frac{1}{\sqrt{3}} \approx 125^{\circ}$ and is repulsive if $55^{\circ}<\theta<125^{\circ}$. It is attractive in other cases. The magnetic interaction potential as well as the attractive and repulsive zones of $\vec{F}_{r}$ are illustrated in Figure 1.

This interaction implies notably that when particles are immersed in a constant and homogeneous magnetic field $\vec{B}$, they self-organize into chains aligned with that field. ${ }^{7,9,10,31-35}$ The exact mechanism of aggregation as well as the distribution of the chains size at thermodynamical equilibrium is still a current research topic, given the observed behavior are not yet fully understood. ${ }^{7,10-12,14}$ This organization is reversible in that meaning that the particles begin to diffuse freely when the magnetic field is switched off, due to the quasi-zero remanence (Figure 3). ${ }^{11,35}$

The self-assembly into chains can be used to create initial conditions where one dimensional diffusion, resulting from the visible random walk of the particles, can be quantitatively studied. Indeed, if the magnetic field is aligned with the $\mathrm{y}$-axis, then all particles from a given chain have a given abscissa $x_{0}$. The initial distribution of the particles center can then be approximated as $\rho\left(\vec{r}, t_{0}\right)=\rho_{0} \delta\left(\vec{r}_{0}\right)$. Actually, the thermal agitation enlarge this distribution implying it is closer from a gaussian curve. But since this is the distribution obtained from an initial delta function, and given the diffusion equation is time invariant, one can just redefine the initial time. This ensures the distribution of the particles 


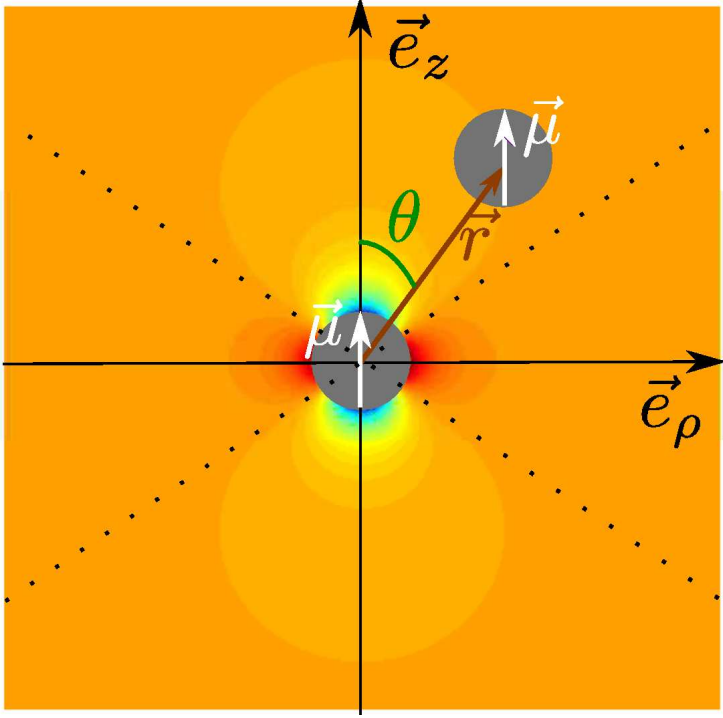

FIG. 1. Graphics of the magnetic interaction potential. The different parameters of $U(r, \theta)$ are illustrated on this graph, and the attractive and repulsive zones of $\vec{F}_{r}$ are delimited by dotted lines. The background colors indicate the value of $U$ at each point, from the blue (largely negative) to the red (largely positive). The orange color denotes a negligible magnetic energy.

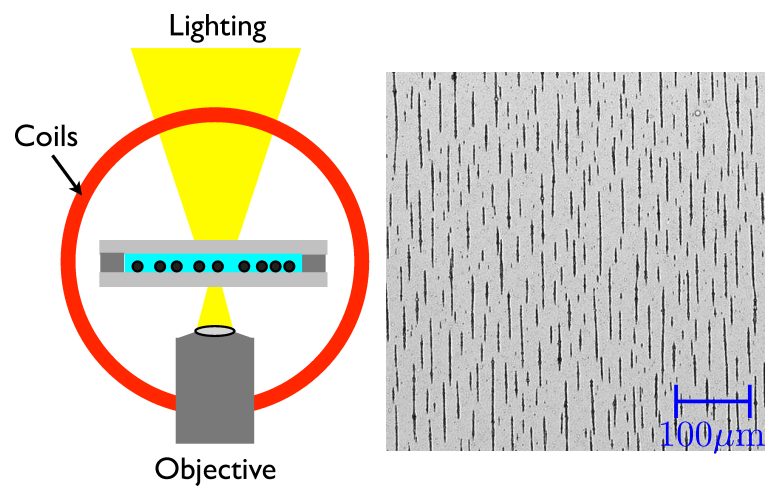

FIG. 2. Sketch of the experimental set-up. A transparent chamber containing the colloidal suspension is placed between two coils generating a constant and homogenous magnetic field $\vec{B}$. The sample is enlightened from the top and observed from the bottom thanks to an inverted microscope. An image obtained during the formation of the chains with a low magnification (10x) is shown on the right.

has then the same evolution as described previously in Eq (3). By analyzing the growth of the FWHM of the particles distribution $W$ as a function of time, one can determine the effective diffusion coefficient of the particles. By doing this for different liquid phases, with varying viscosity, one can also verifies the robustness of the Stokes-Einstein relation by verifying the trend $D \propto \frac{1}{\eta}$. Next section details an experimental protocol to achieve these goals.

\section{METHODS}

A sketch of the experimental set-up designed to study the diffusive disintegration of those chains is presented in the Figure 2. The experiments were performed with superpara- magnetic microspheres dispersed in water (Estapor ${ }^{\circledR}$ M1$070 / 60)$, with a volumic fraction of $\phi=210^{-3}$. A $1 \mu \mathrm{l}$ drop of the suspension is placed inside a cylindrical chamber of diameter $D=5 \mathrm{~mm}$ and thickness $h=50 \mu \mathrm{m}$. The chamber is formed by two parallel glass plates. The first glass plate is covered with a $50 \mu \mathrm{m}$ layer of epoxy at the exemption of a circular region (commercial glasses available, for instance, by Thermo Scientific or Fisher Scientific). A $1 \mu \mathrm{l}$ droplet of suspension is placed inside this region. Afterward, the second glass plate is placed on the first one. A small quantity of low viscosity silicon oil is placed on the epoxy to asses the watertightness of the chamber. The suspension is observed from the bottom with a 40x magnification. A constant and homogeneous magnetic field $B$ is applied by sending a constant current in surrounding coils. When chains are formed, the camera is focused on a long and isolated chain. The current in the coils is then removed to observe the free diffusion of the particles. Images are recorded at the rate of one frame per second, and the field direction is chosen to create chains parallel to one border of the image. An illustrative video of this process is available as supplementary material. ${ }^{41}$

Primary images typically contain about ten chains of superparamagnetic colloids. To study the diffusion of the particles, the first step is then to select one chain and crop the obtained image sequence around that chain (which is the simplest way to define an area of interest for the image analysis and is really straightforward with ImageJ). Once this is done, the distribution of particles can be found by a simple image analysis method we suggest to give as a Matlab coding exercise to students with the following guidelines. The first step is binarizing the image (Most programs have a built-in function able to calculate Otsu's threshold value, which minimize the sum of the intensities variance inside white pixels on one hand and black on the other hand ${ }^{42}$ ). Then, the distribution of particles along the axis perpendicular to the chain can be found by averaging the intensity of the binary image along the axis parallel to the chain, if the particles are white and the background is black. (Clue: this can be done in a single Matlab command, provided the chain is parallel to one side of the picture.) This gives the blue functions in the bottom of the Figure 3. The width $W$ of this experimental distribution can be assessed by the FWHM of a Gaussian fit (which students can perform in Matlab or with any other data analysis software). By plotting this width $W$ as a function of time $t$, one can then estimate the diffusion coefficient $D$ from another fit, with the law $W=4 \sqrt{\ln (2) D\left(t-t_{0}\right)}$, where $D$ and $t_{0}$ are then fitting parameters. ImageJ guidelines and macro, performing both the image analysis and the required fits, are provided as supplementary material as well as a more evolved example of Matlab code. ${ }^{41}$

Since several chains are available from only one image sequence, this image analysis can be performed several times on different chains. This will provide a distribution of diffusion coefficient, whose standard deviation provides an experimental error for this coefficient. For initial chains containing around hundred particles, the typical relative error should be about $10 \%$ as suggested by Poisson law since we actually count the particles in the image analysis.

By repeating this process for surrounding liquids having various viscosity, the validity of the Stokes-Einstein relation can be assessed by fitting the measured diffusion coefficients as a function of the viscosity $D=\frac{C}{\eta}$, with $C$ a fit coefficient 


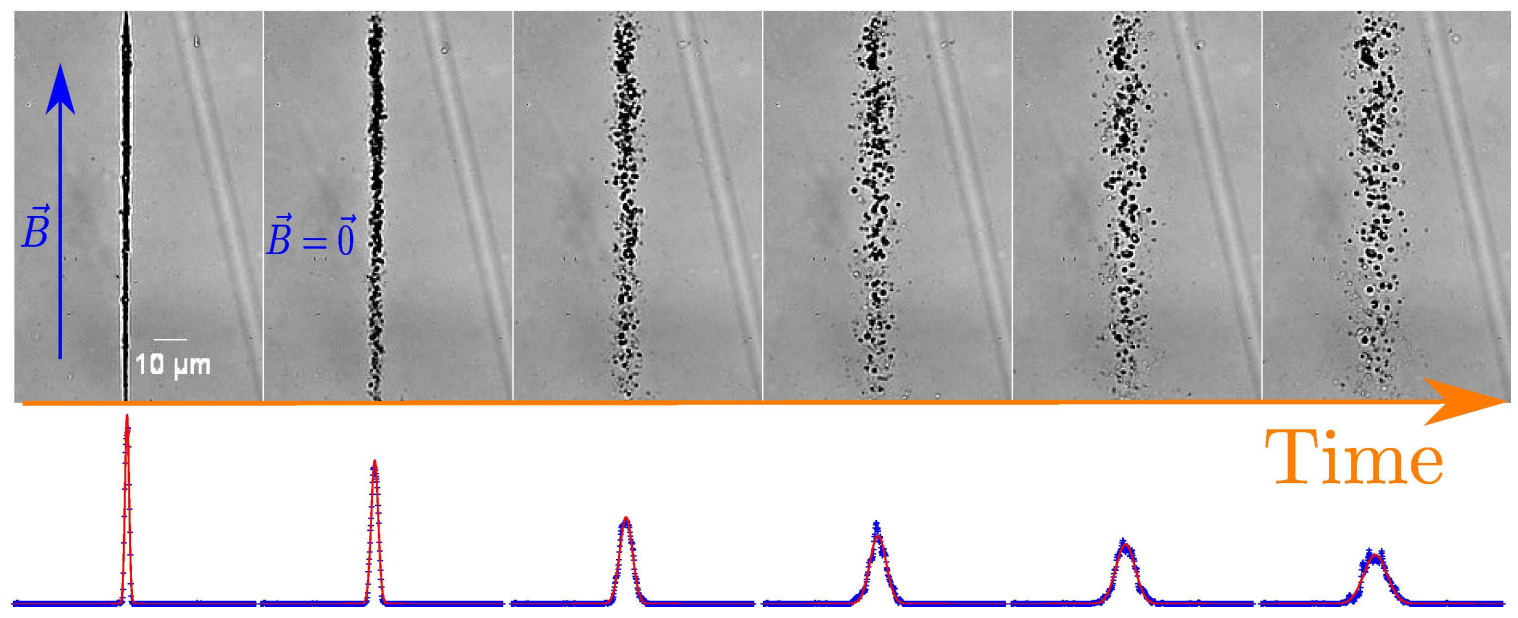

FIG. 3. Experimental illustration of particles diffusion from a chain in water when external magnetic field $\vec{B}$ is switched off. First picture is taken just before the field is suppressed, and there is $2 \mathrm{~s}$ between each image. In blue, the distribution of particles along the $x$-axis is shown for each image, and adjusted by a red Gaussian curve. The units are arbitrary. The width of the distribution increases as time elapses.

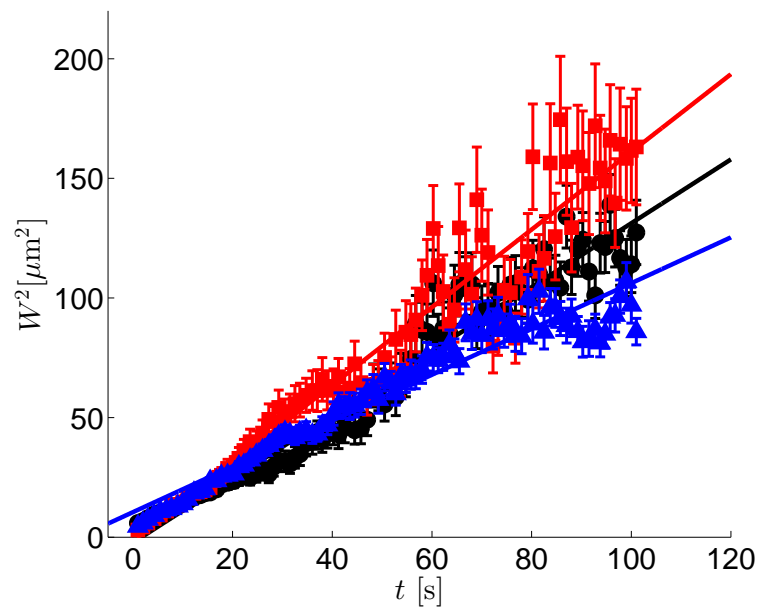

FIG. 4. Evolution of the sqaure of the FWHM $W$ of the Gaussian obtained by fitting experimental data along time $t$ (dots with error bars), for a mixture of glycerol and water containing $40 \%$ in volume of glycerol $(\eta=4.8 \mathrm{mPa} s)$. The evolutions obtained for three different chains have been plotted (each symbol, circle, square or triangle, is related to a specific chain). The data have been fitted with the relation $W=4 \sqrt{\ln (2) D\left(t-t_{0}\right)} \Leftrightarrow W^{2}=$ $16 \ln (2) D\left(t-t_{0}\right)$ (solid lines).

whose value is theoretically given by the mean value of $\frac{k_{B} T}{6 \pi R}$ from the Stokes-Einstein relation. We then propose to perform the process described herein above for different mixture of glycerol and water. The viscosity of such mixture can be calculated or found in tables ${ }^{43}$ but this property can be verified with a rheometer for educative purposes. We always obtained value agreeing with the tables within $5 \%$ variation, consistent with the errors in the measurements required to do the mixture. Detailed results are described in the next section.

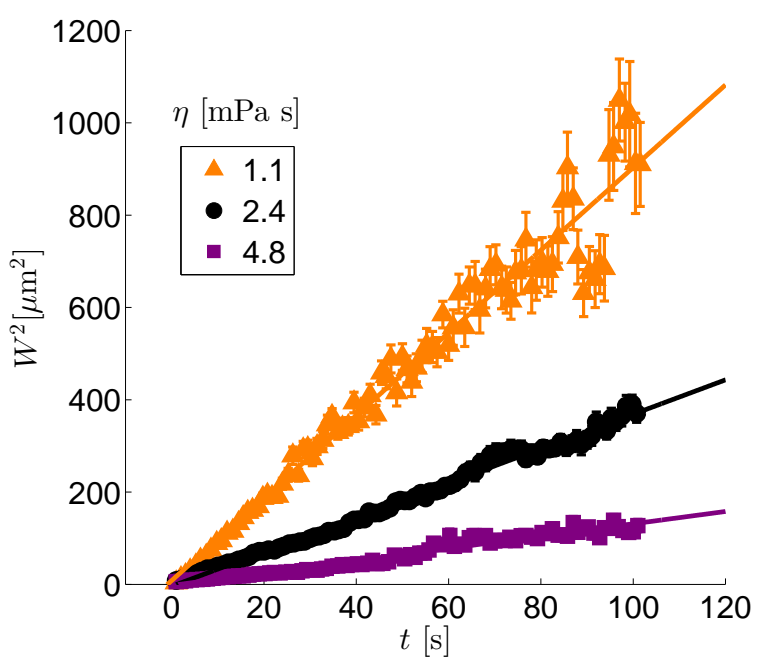

FIG. 5. Typical evolutions of the square of the FWHM $W$ of the Gaussian obtained by fitting experimental data along time $t$ (dots with error bars), for three different mixture of glycerol and water. The viscosity has been measured with a classical rheometer and is consistent with the various concentrations, respectively $6 \%$, $25 \%$ and $40 \%$ of glycerol in volume. The evolutions obtained for three different chains have been plotted (each symbol, circle, square or triangle, is related to a specific chain). The data have been fitted with the relation $W=4 \sqrt{\ln (2) D\left(t-t_{0}\right)} \Leftrightarrow W^{2}=$ $16 \ln (2) D\left(t-t_{0}\right)$ (solid lines).

\section{RESULTS AND DISCUSSION}

Typical evolutions of the FWHM $W$ of the Gaussian distribution, given by the image analysis protocol introduced previously, are represented in the Figures 4 and 5. For clarity purposes, only three chains have been pictured, but the analysis was done with five chains for each glycerol and water mixture. Gathering all the data gives the diffusion coefficients shown in the Figure 6. From those results, we can clearly observe the predicted behavior of $D \propto \frac{1}{\eta}$ from the Equation (2).

Those measurement can be compared more accurately to 
TABLE I. Numerical results and predictions

\begin{tabular}{ccccc}
\hline \hline$\eta[\mathrm{mPas}]$ & $\langle D\rangle\left[\mu \mathrm{m}^{2} / \mathrm{s}\right]$ & $\sigma_{D}\left[\mu \mathrm{m}^{2} / \mathrm{s}\right]$ & $D\left(\left\langle\frac{1}{R}\right\rangle\right)\left[\mu \mathrm{m}^{2} / \mathrm{s}\right]$ & $D\left(\sqrt{\left\langle\frac{1}{R^{2}}\right\rangle}\right)\left[\mu \mathrm{m}^{2} / \mathrm{s}\right]$ \\
\hline 1 & $5.610^{-1}$ & $0.410^{-1}$ & $3.910^{-1}$ & $6.510^{-1}$ \\
1.1 & $5.810^{-1}$ & $0.610^{-1}$ & $3.610^{-1}$ & $5.910^{-1}$ \\
1.2 & $6.010^{-1}$ & $2.10^{-1}$ & $3.310^{-1}$ & $5.410^{-1}$ \\
1.5 & $4.210^{-1}$ & $0.610^{-1}$ & $2.610^{-1}$ & $4.410^{-1}$ \\
2.4 & $3.310^{-1}$ & $0.210^{-1}$ & $1.610^{-1}$ & $2.710^{-1}$ \\
4.8 & $1.110^{-1}$ & $0.210^{-1}$ & $0.810^{-1}$ & $1.410^{-1}$ \\
6.3 & $1.210^{-1}$ & $0.110^{-1}$ & $0.610^{-1}$ & $1.010^{-1}$ \\
8.4 & $7.210^{-2}$ & $0.810^{-2}$ & $4.710^{-2}$ & $7.810^{-2}$ \\
11.5 & $5.710^{-2}$ & $0.410^{-2}$ & $3.410^{-2}$ & $5.710^{-2}$
\end{tabular}

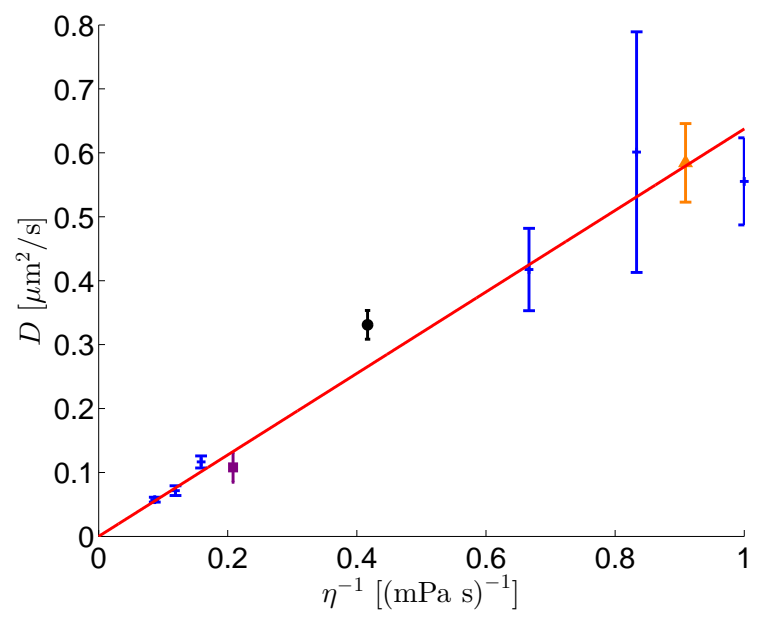

FIG. 6. Diffusion coefficients $D$ obtained for mixtures with different viscosities $\eta$. The points are the data, fitted with a law $D=\frac{C}{\eta}$ (solid line) as predicted by the Stokes-Einstein relation (2). The points in different colors represent the sets of data containing the curves from the figure 5 with the same color. The fit is characterized by a coefficient of determination $R^{2}=0.96$ and the parameter $C=610^{-16} \pm 110^{-16} \mathrm{~kg} \mathrm{~m} \mathrm{~s}^{-2}$.

the Stokes-Einstein relation (2), if the distribution of the radius $R$ of the particles is known. But actually, such a comparison requires a choice of characteristic radius. Indeed, our results summarized in the Table I, along with two theoretical predictions. The size of the particles can be determined through classical image analysis of microscopic photographs. For the same particles as previously, we obtained a wide distribution characterized by a mean value of the inverse radius $\left\langle\frac{1}{R}\right\rangle=1.710^{6} \mathrm{~m}^{-1}$. According to that distribution and the Stokes-Einstein relation, at room temperature $T=300 \mathrm{~K}$, one might think that the predicted value of the diffusion coefficient $D=\frac{k_{B} T}{6 \pi \eta}\left\langle\frac{1}{R}\right\rangle$. The values obtained from this relation have been labelled $D\left(\left\langle\frac{1}{R}\right\rangle\right)$ in the Table I. One can clearly observe that those theoretical values systematically underestimate the diffusion coefficient. This offset comes from the determination of the effective mean radius of the particles. Indeed, the distribution of the particle's radius is very wide and there are many ways to determine a typical inverse radius from this sample. Actually, any quantity which can be expressed as $R_{i, j} \equiv\left(\frac{\left\langle R^{i}\right\rangle}{\left\langle R^{j}\right\rangle}\right)^{\frac{1}{i-j}},\{i, j\} \in \mathbb{Z}$ can be such a typical radius. ${ }^{44}$ However, the viscous forces act on the surface of the particles. The drag, which is one of the main features leading to the Stokes-Einstein relation, is then a surfacedriven effect. Therefore it is more consistent to use an inverse radius derived from the average of the inverse of the surface $\left(\propto R^{2}\right)$ which can be given by $R_{-2,0}=2.910^{6} \mathrm{~m}^{-1}$, so that the effective value of the diffusion coefficient is given by $D\left(\sqrt{\left\langle\frac{1}{R^{2}}\right\rangle}\right)=\frac{k_{B} T}{6 \pi \eta} \sqrt{\left\langle\frac{1}{R^{2}}\right\rangle}$. The values given by this expression are also listed in the Table I. The experimental data oscillate around those values without any systematic offset. Moreover the predicted value of the fit parameter $C=$ $\frac{k_{B} T}{6 \pi} \sqrt{\left\langle\frac{1}{R^{2}}\right\rangle}=6.510^{-16} \mathrm{~kg} \mathrm{~m} \mathrm{~s}^{-2}$ is then lying in the error bars of our fitting value of $C=610^{-16} \pm 110^{-16} \mathrm{~kg} \mathrm{~m} \mathrm{~s}^{-2}$. This enhances how important it is to consider the right characteristic length and how the width of a parameter's distribution can have a strong influence on theoretical predictions. However, we can conclude that the value we obtained by our method of measurement is then consistent with the distribution of size of the particles and the Stokes-Einstein relation.

\section{CONCLUSION}

The present paper has described a simple but original experiment suitable to introduce the diffusion of particles as a result from their random walk due to Brownian motion, which is a standard topic of statistical physics to students. Moreover, this experiment involves superparamagnetic colloids and may be used to show them the relation between this current area of research and standard topics of statistical physics. This experiment also offers an opportunity to introduce some basic image analysis techniques as experimental physics skills as well as some examples of experimental fits to compare with theoretical laws.

\section{ACKNOWLEDGMENTS}

$\mathrm{AD}$ is financially supported by an FNRS grant as research fellow. J.F. thanks the FNRS and Alexander Von Humboldt Foundation. This work was financially supported by the FNRS (Grant PDR T.0043.14) and by the University of Liège (Starting Grant C-13/88). The authors thank YvesÉric Corbisier from the physics department of the University of Liège for technical support. 
* alexis.darras@ulg.ac.be

1 B. J. Krishnatreya et al., American Journal of Physics 82, 23 (2014).

2 S. Harris, American Journal of Physics 44, 291 (1976).

3 P. Nakroshis, M. Amoroso, J. Legere, and C. Smith, American Journal of Physics (2003).

4 M. A. Catipovic, P. M. Tyler, J. G. Trapani, and A. R. Carter, American Journal of Physics 81, 485 (2013).

${ }^{5}$ K. Ghosh, K. A. Dill, M. M. Inamdar, E. Seitaridou, and R. Phillips, American journal of physics 74, 123 (2006).

6 B. Brazzle, American Journal of Physics 81, 823 (2013).

7 J. Faraudo, J. S. Andreu, and J. Camacho, Soft Matter 9, 6654 (2013).

8 J. Faraudo and J. Camacho, Colloid Polym. Sci. 288, 207 (2010).

9 J. S. Andreu, J. Camacho, and J. Faraudo, Soft Matter 7, 2336 (2011).

10 J. H. Promislow, A. P. Gast, and M. Fermigier, J. Chem. Phys. 102, 5492 (1995).

11 P. Domínguez-García, J. Pastor, and M. Rubio, Eur. Phys. J. E 34, 1 (2011).

12 P. Domínguez-García, S. Melle, J. Pastor, and M. Rubio, Phys. Rev. E 76, 051403 (2007).

13 M. Fermigier and A. P. Gast, Journal of colloid and interface science 154, 522 (1992).

14 A. Darras, J. Fiscina, M. Pakpour, N. Vandewalle, and G. Lumay, The European Physical Journal E 39, 1 (2016).

15 C. T. Yavuz et al., Science 314, 964 (2006).

16 K. M. Krishnan, IEEE Trans. Magn. 46, 2523 (2010).

17 J. L. Corchero and A. Villaverde, Trends Biotechnol. 27, 468 (2009).

18 U. Jeong, X. Teng, Y. Wang, H. Yang, and Y. Xia, Adv. Mater. 19, 33 (2007).

19 P. Liu, J. W. De Folter, A. V. Petukhov, and A. P. Philipse, Soft Matter 11, 6201 (2015).

20 H. Carstensen, V. Kapaklis, and M. Wolff, Phys. Rev. E 92, 012303 (2015).

21 F. Martinez-Pedrero, A. Ortiz-Ambriz, I. Pagonabarraga, and P. Tierno, Phys. Rev. Lett. 115, 138301 (2015).

${ }^{22}$ F. Martinez-Pedrero and P. Tierno, Phys. Rev. Applied 3, 051003 (2015).
${ }^{23}$ R. M. Erb, H. S. Son, B. Samanta, V. M. Rotello, and B. B Yellen, Nature 457, 999 (2009).

24 M. Llera, J. Codnia, and G. A. Jorge, J. Magn. Magn. Matter 384, 93 (2015).

${ }^{25}$ K. Muller et al., Langmuir 30, 5088 (2014).

26 J. W. Tavacoli et al., Soft Matter 9, 9103 (2013).

27 G. Lumay, N. Obara, F. Weyer, and N. Vandewalle, Soft Matter 9, 2420 (2013).

28 G. Grosjean et al., Scientific reports 5 (2015).

29 M. Pichumani and W. González-Viñas, Magnetohydrodynamics 47, 191 (2011).

30 P. Pearle et al., American Journal of Physics 78, 1278 (2010).

31 S. Fraden, A. J. Hurd, and R. B. Meyer, Phys. Rev. Lett. 63, 2373 (1989).

32 M. Kolb, Phys. Rev. Lett. 53, 1653 (1984).

33 F. Martínez-Pedrero, M. Tirado-Miranda, A. Schmitt, and J. Callejas-Fernández, Phys. Rev. E 76, 011405 (2007).

34 S. Miyazima, P. Meakin, and F. Family, Phys. Rev. A 36, 1421 (1987).

35 G. P. Gajula, M. T. Neves-Petersen, and S. B. Petersen, Appl. Phys. Lett. 97, 103103 (2010).

${ }^{36}$ K. S. Khalil et al., Nat. Commun. 3, 794 (2012).

37 Y. Gurevich, Y. Mankov, and R. Khlebopros, Dokl. Phys. 11, 478 (2013).

38 F. Martinez-Pedrero and P. Tierno, Phys. Rev. Applied 3, 051003 (2015).

39 H. Carstensen, V. Kapaklis, and M. Wolff, Phys. Rev. E 92, 012303 (2015).

${ }^{40}$ K. H. Taylor, The influence of magnetic cohesion on the stability of granular slopes, $\mathrm{PhD}$ thesis, University of Nottingham, 2009.

41 [See supplementary materials at [URL will be inserted by AIP] for Matlab script for image analysis as well as ImageJ macro and guidelines. An illustrative video of the experimental protocol is available too.].

42 N. Otsu, Automatica 11, 23 (1975).

43 N.-S. Cheng, Industrial \& engineering chemistry research 47, 3285 (2008).

44 T. Cosgrove, Colloid science: principles, methods and applications (John Wiley \& Sons, 2010). 